\title{
p.[G576S; E689K]: pathogenic combination or polymorphism in Pompe disease?
}

\author{
Marian A Kroos ${ }^{1}$, Reinier A Mullaart ${ }^{2}$, Laura Van Vliet ${ }^{1}$, Robert J Pomponio ${ }^{3}$, \\ Hernan Amartino ${ }^{4}$, Edwin H Kolodny ${ }^{5}$, Gregory M Pastores ${ }^{5}$, Ron A Wevers ${ }^{2}$, \\ Ans T Van der Ploeg ${ }^{1}$, Dicky JJ Halley ${ }^{1}$ and Arnold JJ Reuser ${ }^{*, 1}$
}

${ }^{1}$ Departments of Clinical Genetics and Pediatrics, Erasmus MC, Rotterdam, The Netherlands; ${ }^{2}$ Department of Pediatrics and Neurology, Radboud University Medical Centre, Nijmegen, The Netherlands; ${ }^{3}$ Clinical Laboratory Science; Pharmacogenetics, Genzyme Corp., Framingham, MA, USA; ${ }^{4}$ Laboratory of Neurochemistry 'Dr Nestor Chamoles', Buenos Aires, Argentina; ${ }^{5}$ Department of Neurology, New York University School of Medicine, New York, USA

We discuss four cases of acid $\alpha$-glucosidase deficiency (EC, 3.2.1.3/20) without evident symptoms of Pompe disease (OMIM No 232300) in individuals of Asian descent. In three cases, the deficiency was associated with homozygosity for the sequence variant c.[1726G $>A ; 2065 G>A]$ in the acid $\alpha$-glucosidase gene $(G A A)$ translating into p.[G576S; E689K]. One of these cases was a patient with profound muscular atrophy, another had cardio-myopathy and the third had no symptoms. The fourth case, the mother of a child with Pompe disease, was compound heterozygote for the GAA sequence variants c.[1726G $>A ; 2065 G>A] /$ c.2338G $>$ A (p.W746X) and had no symptoms either. Further investigations revealed that c. [1726A; 2065A] is a common GAA allele in the Japanese and Chinese populations. Our limited study predicts that approximately $4 \%$ of individuals in these populations are homozygote c.[1726A; 2065A]. The height of this figure in contrast to the rarity of Pompe disease in Asian populations and the clinical history of the cases described in this paper virtually exclude that homozygosity for c.[1726A; 2065A] causes Pompe disease. As c. $[1726 \mathrm{~A} ; 2065 \mathrm{~A}]$ homozygotes have been observed with similarly low acid $\alpha$-glucosidase activity as some patients with Pompe disease, we caution they may present as false positives in newborn screening programs especially in Asian populations.

European Journal of Human Genetics (2008) 16, 875-879; doi:10.1038/ejhg.2008.34; published online 27 February 2008

Keywords: acid maltase; glucosidase; glycogen storage disease; lysosomal; newborn screening

\section{Introduction}

Pompe disease also known as glycogen storage disease type II or acid maltase deficiency (OMIM No 232300) is a rare, autosomal recessive, metabolic disorder. The disease is caused by pathogenic sequence variations in the acid $\alpha$-glucosidase gene $(G A A)$. Acid $\alpha$-glucosidase is required for

*Correspondence: Dr AJJ Reuser, Department of Clinical Genetics, Erasmus MC, P.O. Box 2040, Rotterdam 3000 CA, The Netherlands. Tel: + 3110 7043153; Fax: + 31107044736 ;

E-mail: a.reuser@erasmusmc.nl

Received 26 September 2007; revised 28 December 2007; accepted 25 January 2008; published online 27 February 2008 the intra-lysosomal degradation of glycogen. Enzyme deficiency leads to lysosomal accumulation of glycogen in many different cell types, but affects primarily the skeletal muscle function. Cardiac muscle involvement is limited to cases wherein the enzyme deficiency is virtually complete. $^{1,2}$ At present, close to 200 GAA sequence variations have been identified (see http: www.pompecenter.nl).

c.1726G > A (p.G576S) is one of two sequence variations that we discovered in both GAA alleles of a Japanese boy presenting with ECG abnormalities, but no muscle weakness (Case 1, previously described by Suzuki et $a l^{3}$ ). Pipo et $a^{4}$ described that this variant reduces the acid 
$\alpha$-glucosidase activity by about half. Apart from being homozygous for c.1726G >A (p.G576S) the patient also was homozygous for c.2065G > A (p.E689K). ${ }^{5}$ As previously reported by Suzuki et $\mathrm{al}^{3}$, the child had moderately low acid $\alpha$-glucosidase activity for the artificial substrate 4-methylumbelliferyl- $\alpha$-D-glucopyranoside (43\% of normal) and markedly reduced activity for the natural substrate glycogen ( $14 \%$ of normal). The $\mathrm{Km}$ of acid $\alpha$-glucosidase for glycogen was fivefold elevated and the amount of acid $\alpha$-glucosidase-protein clearly reduced. The relationship between the GAA genotype, the acid $\alpha$-glucosidase deficiency and the ECG abnormality remained enigmatic. p.K689 characterizes the GAA4 allozyme which was formerly found in the Chinese population with a frequency of 0.28 compared with the more common GAA1 allele (p.E689). ${ }^{6-8}$

Recently, we became involved in the diagnosis of a boy with congenital arthrogryposis (Case 2). Measurement of a series of lysosomal enzyme activities in leukocytes, cultured skin fibroblasts and muscle led to the serendipitous finding of acid $\alpha$-glucosidase deficiency while subsequent analysis of the $G A A$ gene revealed homozygosity for the combination p.[G576S; E689K].

During the diagnostic work-up, a third case of homozygosity for p.[G576S; E689K] came to our attention (Case 3). It concerned a healthy Japanese woman who had donated blood to serve as control sample in a newborn screening test for Pompe disease, but came out as false positive.

In the fourth case (Case 4), we were confronted with a difficult to interpret familial situation wherein the healthy, 25-year-old, Chinese mother of a child with classic infantile Pompe disease was identified as compound heterozygote: p.W746X/p.[G576S; E689K].

As the c. $[1726 \mathrm{G}>\mathrm{A} ; 2065 \mathrm{G}>\mathrm{A}]$ allele translating into p.[G576S; E689K] played a role in all of these four cases we analyzed the effect of both mutations, singly and in combination. The results are discussed in relation to the incidence of p.[G576S; E689K] in Asian populations and newborn screening for Pompe disease.

\section{Materials and methods Case reports}

Case 1: A previous report of a 12-year-old Japanese boy presenting with ECG abnormalities, but no muscle weakness (Case 1 in Table 1). ${ }^{3,9}$ Within the next 2 years, he developed congestive heart failure. The child had moderately low acid $\alpha$-glucosidase activity for the artificial substrate 4-methylumbelliferyl- $\alpha$-D-glucopyranoside (43\% of normal) and markedly reduced activity for glycogen (14\% of normal). The amount of acid $\alpha$-glucosidase was clearly reduced and the $\mathrm{Km}$ for the natural substrate glycogen was fivefold elevated. The relationship between the acid $\alpha$-glucosidase deficiency and the ECG abnormality remained enigmatic.

Case 2: A boy of Chinese descent presented at birth with multiple contractures. Pregnancy and birth, both had a complicated course (polyhydramnios and placental abruption). Despite sufficient Apgar-scores (eight after $5 \mathrm{~min}$ ) he suffered many complications during his first year of life including, at the age of 7 months, acquired but unexplained bilateral subdural effusion. Now, at the age of 14 years, he presents with multiple contractures, profound generalized muscle weakness and atrophy, depressed stretch reflexes, polyminimyoclonus and tongue fibrillations. He has no sign of brain dysfunction. Muscle biopsy at the age of 2 months showed groupwise atrophy, locally hypertrophic fibers and excessive interfascicular connective tissue. At the age of 6 years, the activities of several lysosomal enzymes were measured as part of the diagnostic workup and acid $\alpha$-glucosidase deficiency was noted. Ancillary tests including $S M N$-analysis, cardiac examination, repeated serum creatine kinase determination and reexamination of the muscle tissue obtained at the age of 2 years for signs of glycogen storage, yielded normal results.

Case 3: This asymptomatic woman was identified as having low acid $\alpha$-glucosidase activity while participating, with informed consent, in a research study to establish the normal range for acid $\alpha$-glucosidase in dried blood spots taken from healthy individuals. This was to serve as the reference population for a newborn screening assay for

Table 1 Acid $\alpha$-glucosidase activities in leukocytes, lymphocytes, cultured fibroblasts and muscle of reported cases

\begin{tabular}{|c|c|c|c|c|c|}
\hline & & Acid $\alpha$-gluc & vity $^{a}$ & & \\
\hline & & Lymphocytes & & Fibroblasts & \\
\hline & Leukocytes glucose & $M U$ & $M U$ & Glucose & Muscle glucose \\
\hline Case 1 & & & $71(43)$ & $172(14)$ & \\
\hline Case 2 & $7 / 20^{\mathrm{b}}(3 / 10)$ & & $39(24)$ & $96(7)$ & $25(31)$ \\
\hline Case 3 & & $7(10)$ & & & \\
\hline
\end{tabular}

${ }^{a}$ The activities were measured with 4-methylumbelliferyl- $\alpha$-D-glucopyranoside and with glycogen as substrates and are expressed in nmoles methylumbelliferone (MU) or nmoles glucose formed per hour per mg protein.

${ }^{\text {b}}$ Two independent assays. The normal ranges were as follows: Glycogen activity in leukocytes: 80-360; MU activity in lymphocytes: 29-96; MU activity in fibroblasts: 80-260; Glycogen activity in fibroblasts: 340-1100; Glycogen activity in muscle: $30-120$. The figures between brackets indicate the activities as percentage of average normal. 
Pompe disease. The woman, 29 years old at the time and of Japanese descent, had no signs of Pompe disease despite having low acid $\alpha$-glucosidase (Case 3 in Table 1). Her serum CK value was normal.

Case 4: is the mother of a child with classic infantile Pompe disease. Her child was previously described as Case A in reference. ${ }^{10}$ The mother self-reported her ethnicity as Chinese. The clinical diagnosis for the child was confirmed, based on the presence of acid $\alpha$-glucosidase deficiency and compound heterozygosity for the pathogenic GAA sequence variations c.2338G $>\mathrm{A}$ and c.1843G $>$ A (p.W746X and p.G615R), found on separate alleles. The mother was a compound heterozygote p.W746X / p.[G576S; E689K]. Data on her acid $\alpha$-glucosidase activity are unfortunately lacking, but she was 25 years old when her physical and neurological examinations were unremarkable.

\section{DNA analysis}

$G A A$ gene analysis was performed with informed consent of the patients as part of the diagnostic procedure. Anonymous DNA samples from 22 healthy Japanese individuals were tested for the presence of c.1726G $>$ A and c.2065G $>$ A. For this purpose, exon 12 and exon 15 were amplified by PCR and sequenced using the following primer sets: Primer set 1 for exon 12: 5'-TGTAAAACGACG GCCAGTGGGAGAAGCTTCCTGGAAAC- $3^{\prime}$ and $5^{\prime}$-CAGGA AACAGCTATGACCCCCAACCTTGTAGGACAGGCT-3'; and primer set 2 for exon 15: 5'-TGTAAAACGACGGCCAGTTG AGAAGTGCAGCTCTCCCG-3' ${ }^{\prime}$ and 5'-CAGGAAACAGCTAT GACCTCCCCCCAGGGCACACATGGCCAC-3'. Mutation specific PCR (ARMS) was performed on DNA samples in which both mutations were found heterozygous to determine whether they were located on the same or on different alleles. The primers used for this purpose were: 5'-TACAACCTGCACAACCT CTACG-3'and 5'-GGCCTGCT GGGCCGGCTC-3'.

\section{Haplotype analysis}

NM_000152.3 was used as reference sequence for the coding regions (http://www.ncbi.nlm.nih.gov/entrez/viewer. fcgi?db $=$ nucleotide\&val $=119393890)$, whereby the ' $A$ ' nucleotide of the ATG codon at nucleotide position 442 constitutes +1 numbering of the cDNA sequence. This ATG codon represents +1 for the amino acid numbering as set forth by the acid $\alpha$-glucosidase pre-protein sequence NP 000143.2 (http://www.ncbi.nlm.nih.gov/entrez/viewer.fcgi? $\mathrm{db}=$ protein\&val $=$ NP_000143.2).

The positions of intronic sequence variations were compared with the genomic contig sequence NT_024871 whereby the 'A' of the ATG start codon is position 481925.11 (http://www.ncbi.nlm.nih.gov/entrez/viewer.fcgi? dispmax $=1 \&$ showndispmax $=1 \&$ val $=$ NT_024871.11\&view $=$ graph\&_from $=472690 \&$ \&sfrom $=480354 \&$ to $=503346$ ).

The three reference sequences used in this study can be found at http://www.pompecenter.nl/position.pdf.

\section{Enzyme activity assay, transfection and immunoblotting \\ Methods used were those described previously. ${ }^{11,12}$}

\begin{abstract}
Results
A boy of Chinese descent suffered many complications from birth on (Case 2). At the age of 6 years, the activities of several lysosomal enzymes were measured as part of the diagnostic workup and acid $\alpha$-glucosidase deficiency was noted (Case 2 in Table 1). Sequence analysis of the exons of the acid GAA revealed three variations when compared with the cDNA reference sequence NM_000152.3 whereby the ' $A$ ' nucleotide of the start codon at nucleotide position 442 constitutes +1 numbering of the sequence: c.1726G $>$ A (p.G576S), c.2065G $>$ A (p.E689K) and c.2133A $>$ G (p.T711T), all homozygous. The first two mutations were previously described in relation to Pompe disease and investigated for their individual effect but not for their combined effect. ${ }^{4,5,9,13,14}$ We introduced these mutations, singly and in combination, into wild type $G A A$ cDNA and studied their effect by transient expression in COS-7 cells. Table 2 shows that c.1726G >A (S576) alone reduces the acid $\alpha$-glucosidase activity by approximately $80 \%$ when compared with the expression of wild-type cDNA c.[1726G; 2065G] (G576; E689), while c.2065G > A (K689) alone leads to approximately $50 \%$ reduction. When
\end{abstract}

Table 2 Activities of the various GAA cDNA constructs transiently expressed in COS-7 cells

\begin{tabular}{lcc}
\hline$G A A-C D N A$ construct & MU & Acid $\alpha$-glucosidase activity ${ }^{\mathrm{a}}$ \\
\hline$[\mathrm{G} 576 ; \mathrm{E} 689](n=4)$ & $100(190-366: 263)$ & $100(640-1098: 1550)$ \\
{$[\mathrm{S} 576](n=5)$} & $18(31-64: 48)$ & $17(144-212: 185)$ \\
{$[\mathrm{K} 689](n=5)$} & $54(90-190: 142)$ & $57(440-842: 622)$ \\
{$[\mathrm{S} 576 ; \mathrm{K} 689](n=9)$} & $23(19-94: 60)$ & $22(23-375: 240)$ \\
\hline
\end{tabular}

${ }^{\text {a }}$ The activities were measured with 4-methylumbelliferyl- $\alpha$-D-glucopyranoside and with glycogen as substrates and are expressed in nmoles methylumbelliferone (MU) or nmoles glucose per hour per mg protein as percentages of the mean activity in cells expressing p.[G576; E689]. The figures between brackets represent the range of activities measured in separate experiments and the mean activity in bold. 
combined, the two mutations c.[1726A; 2065A] (S576; K689) have the same effect as c.1726G >A (S576) alone and result in a degree of acid $\alpha$-glucosidase deficiency that can be found in children and adults with Pompe disease. ${ }^{9}$ Figure 1 illustrates that the amount of acid $\alpha$-glucosidaseprotein is proportionally reduced. The results obtained by these analyses were in accordance with the activities measured in the cells and tissue samples of the patient (Case 2 in Table 1).

Two more cases of p.[G576S; E689K] homozygosity associated with remarkably low acid $\alpha$-glucosidase activity came to our attention. Case 1 in Table 1 is the patient first described by Suzuki et $\mathrm{al}^{3}$ and recently referred to by Tajima et $a l^{5}$. Case 3 came to light in a series of DNA samples from healthy individuals. The woman, 29 years old, was of Japanese descent and had no signs of Pompe disease, but a profound deficiency of acid $\alpha$-glucosidase in a bloodspot test. The deficiency was confirmed by measuring the acid $\alpha$-glucosidase activity in peripheral lymphocytes (Case 3 in Table 1). Her serum CK value was within normal limits.

In a fourth case (Case 4) we encountered the combination p.[G576S; E689K]/p.W746X on the separate alleles of the mother of a child with classic infantile Pompe disease, in a family self-identified as Chinese. Unfortunately, we did not obtain a blood sample or other tissue specimen from the mother to assay her acid $\alpha$-glucosidase activity, but she was 25 years old when she had a physical examination and not noticed to have any clinical sign of Pompe disease even after 2 years of follow up observation.

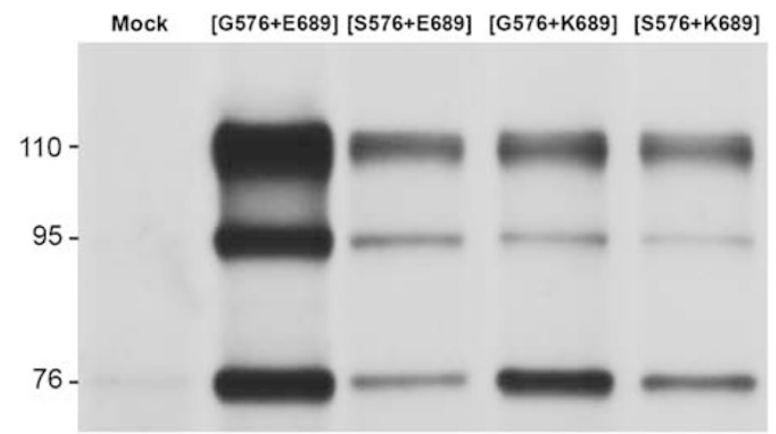

Figure 1 Synthesis and maturation of wild type and mutant forms of acid $\alpha$-glucosidase in transiently transfected COS- 7 cells. COS- 7 cells were transfected with wildtype, p.G576S, p.E689K and p.[G576S; E689K] CDNA constructs of the GAA gene and harvested 3 days later. Cell homogenates ( $50 \mu \mathrm{g}$ of cellular protein per lane) were made and subjected to polyacrylamide gel electrophoresis in the presence of sodium dodecylsulphate (SDS-PAGE) to separate the precursor $(110 \mathrm{kDa})$, the intermediate $(95 \mathrm{kDa})$ and the lysosomal $(76 \mathrm{kDa})$ forms of acid $\alpha$-glucosidase. These were visualized by immunoblotting using rabbit polyclonal antibodies raised against the human placental enzyme in combination with $\mathrm{ECL}^{\mathrm{TM}}$ western blotting detection reagents (Amersham Biosciences, Piscataway, NJ, USA). The mocktransfected cells were treated with empty vector.

\section{Discussion}

The p. E689K substitution was originally identified as the molecular basis of the GAA4 allozyme of acid $\alpha$-glucosidase known to be frequent in the Asian population. ${ }^{6-8}$ Transient expression of c.2065G $>$ A cDNA (p.E689K) was reported to result in $72-125 \%$ of normal acid $\alpha$-glucosidase activity. ${ }^{13,14}$ In another publication transient expression of c.1726G > A cDNA (p.G576S) was reported to result in 50\% of normal activity. ${ }^{4}$ In our expression system, the activity of each of the two sequence variations came out lower. This may be due to the different procedure. The combined effect of both sequence variants was not previously investigated and was found in this study to be the same as of c. $1726 \mathrm{G}>\mathrm{A}$ alone. The combination leads to roughly 60$80 \%$ 'loss' of acid $\alpha$-glucosidase activity, and this figure matches the activities that were measured in tissue samples of Case 1, 2 and 3 (Table 1).,

We noticed that the few reported cases of p.G576S, including the ones presented here, were all Asian and searched for the frequencies of sequence variations in Asian and non-Asian populations. The literature data are summarized in Table 3 (NCBI sample sets) and confirm that substitution p.E689K is frequent in the Japanese and Chinese populations, but quite rare in the European and sub-Saharan/African populations. The same holds for substitution p.G576S. Notably, it seems that p.K689 can occur in isolation, but p.S576 is invariably found in combination with p.K689 (Table 3). We obtained further support for the hypothesis that p.S576 solely occurs in allelic conjunction with p.K689, by sequencing the $G A A$ gene of 22 healthy Japanese individuals. None of the 44 alleles had haplotype c.[1726A; 2065G] coding for p.[S576; E689], but two had haplotype c.[1726G; 2065A] coding for p.[G576; K689]. One of the 22 Japanese individuals was homozygote p.[S576; K689] and eight individuals were heterozygote at both positions. In seven of these eight cases we could confirm allelic association of c.[1726A; 2065A]. In one case the allele-specific PCR failed, because of impurity and shortage of DNA. The allele frequencies are listed in Table 3 (Erasmus MC sample set). In summary, both p.G576S and p.E689K are frequent GAA sequence variations in Asian countries that mostly occur in allelic conjunction. The estimated frequency of the p.[S576; K689] allele is less than 0.01 in the European and subSaharan/African populations but as high as 0.2 in Asian populations (Table 3).

Collectively, the findings suggest that the complex of clinical symptoms in Case 2 (Chinese descent) and the cardiac arrhythmia in Case 1 (Japanese descent) are unrelated to the remarkably low acid $\alpha$-glucosidase activity caused by homozygosity for GAA genotype c.[1726G $>\mathrm{A}$; $2065 \mathrm{G}>\mathrm{A}$ ] translating into p.[G576S; E689K]. This is supported by the fact that a third individual with a similarly low activity and the same genotype (Japanese, Case 3) did not have any sign of disease. Even the mother 
Table 3 Kind and frequency of GAA alleles encoding p.G576 or p.S576, and p.E689 or p.K689 in different populations

\begin{tabular}{|c|c|c|c|c|c|}
\hline \multirow{2}{*}{ Number of alleles } & \multicolumn{5}{|c|}{ Populations } \\
\hline & Japanese $^{\mathrm{a}}$ & Chinese $^{\mathrm{a}}$ & European $^{\mathrm{a}}$ & Sub-Saharan/African ${ }^{\mathrm{a}}$ & Japanese $^{\mathrm{b}}$ \\
\hline $\begin{array}{l}{[\mathrm{G}+\mathrm{E}]} \\
{[\mathrm{S}+\mathrm{E}]} \\
{[\mathrm{G}+\mathrm{K}]} \\
{[\mathrm{S}+\mathrm{K}]} \\
\mathrm{G} / \mathrm{S}+\mathrm{E} / \mathbf{K}^{\mathrm{c}} \\
\text { Total }\end{array}$ & $\begin{array}{c}49(56) \\
0(0) \\
8(9) \\
7(8) \\
24^{\mathrm{c}}(27) \\
88(100)\end{array}$ & $\begin{array}{c}53(59) \\
0(0) \\
9(10) \\
4(4) \\
24^{\mathrm{c}}(27) \\
90(100)\end{array}$ & $\begin{array}{c}176(98) \\
0(0) \\
4(2) \\
0(0) \\
0(0) \\
180(100)\end{array}$ & $\begin{array}{c}178(100) \\
0(0) \\
0(0) \\
0(0) \\
0(0) \\
178(100)\end{array}$ & $\begin{array}{c}31(70) \\
0(0) \\
2(5) \\
9(20) \\
2(5) \\
44(100)\end{array}$ \\
\hline
\end{tabular}

${ }^{a} \mathrm{NCBI}$ sample sets.

${ }^{\mathrm{b}}$ Erasmus MC sample set.

'The mutations could not be assigned to individual alleles. The figures indicate how often each allele was encountered. The percentage is given in brackets. Given the rarity of haplotype $[\mathrm{S}+\mathrm{E}]$, it seems justified to divide the number of 24 undefined $\mathrm{G} / \mathrm{S}+\mathrm{E} / \mathrm{K}$ alleles over $[\mathrm{G}+\mathrm{E}]$ en $[\mathrm{S}+\mathrm{K}]$. This would change the figures in: $[G+E]=69 \%\left(\right.$ Japanese $\left.^{a}\right), 72 \%\left(\right.$ Chinese $\left.^{a}\right)$ and 73\% (Japanese $\left.{ }^{b}\right) ;[S+K]=22 \%\left(J a p a n e s e^{a}\right), 18 \%\left(C^{2}\right.$,

of an affected child with potentially higher risk genotype p.[G576S; E689K]/p.W746X (Chinese, Case 4), did not manifest any signs of Pompe disease at the age of 25 years. From a theoretical point of view, if homozygosity for p.[G576S; E689K] would lead to Pompe disease or clinical symptoms of other nature, the frequency of affected individuals in Asian countries would be very high based on the allele frequencies. This is not the case. There is just one report of a Pakistani patient with clinically confirmed classic infantile Pompe disease who was homozygous p.[G576S; E689K]. But, this patient also was homozygous for the non-sense mutation c.C118T (R40X) and had much less residual activity than the cases described in the current report. ${ }^{15}$ Healthy individuals with one p.[G576S; E689K] allele and one 'normal' GAA allele are certainly not at risk for developing Pompe disease. But it cannot be excluded that compound heterozygotes with one p.[G576S; E689K] allele and one $G A A$ allele with a pathogenic mutation develop 'Pompe disease like' symptoms late in life due to their predictably very low acid $\alpha$-glucosidase activity, as in Case 4 . Follow-up of these individuals will teach about the real genetic risk and allow for timely intervention as well as appropriate genetic counseling and expectation of possible disease progression, or facets thereof.

The predicted high frequency of p.[G576S; E689K] homozygosity in Asian populations (approximately 0.04, Table 3) has potential consequences for newborn screening programs. Since homozygotes can have an estimated $60-80 \%$ reduction of acid $\alpha$-glucosidase activity compared to average normal some of them may manifest as false positives, like the woman in our study (Case 3).

\section{Acknowledgements}

This research was funded in part by an unrestricted grant from Genzyme Corporation, Cambridge, MA, USA. Tom de Vries Lentsch kindly prepared the illustrations.

\section{References}

1 Hirschhorn R, Reuser AJJ: Glycogen Storage Disease Type II (GSDII); in Scriver CR, Beaudet AL, Sly WS, Valle D (eds):: The
Metabolic and Molecular Bases of Inherited Disease. NY: McGrawHill, 2001, pp 3389-3420.

2 Engel AG, Hirschhorn R, Huie ML: Acid maltase deficiency; in Engel AG, Franzini-Armstrong C (eds): Myology. NY: McGraw-Hill, 2004, pp 1559-1586.

3 Suzuki Y, Tsuji A, Omura $\mathrm{K}$ et al: Km mutant of acid alphaglucosidase in a case of cardiomyopathy without signs of skeletal muscle involvement. Clin Genet 1988; 33: 376-385.

4 Pipo JR, Feng JH, Yamamoto T et al: New GAA mutations in Japanese patients with GSDII (pompe disease). Pediatr Neurol 2003; 29: 284-287.

5 Tajima Y, Matsuzawa F, Aikawa S et al: Structural and biochemical studies on Pompe disease and a 'pseudodeficiency of acid alpha-glucosidase'. J Hum Genet 2007; 52: 898-906.

6 Nickel BE, McAlpine PJ: Extension of human acid alpha-glucosidase polymorphism by isoelectric focusing in polyacrylamide gel. Ann Hum Genet 1982; 46: 97-103.

7 Huie ML, Menaker M, McAlpine PJ, Hirschhorn R: Identification of an E689K substitcas the molecular basis of the human acid $\alpha$-glucosidase type 4 allozyme (GAA*4). Ann Hum Genet 1996; 60: $365-368$.

8 Shieh JJ, Lin CY: Frequent mutation in Chinese patients with infantile type of II GSD in Taiwan: evidence for a founder effect. Hum Mutat 1998; 11: 306-312.

9 Reuser AJJ, Kroos MA, Hermans MMP et al: Glycogenosis type II (acid maltase deficiency). Muscle Nerve 1995; 3: S61-S69.

10 Kishnani PS, Nicolino M, Voit $\mathrm{T}$ et al: Chinese hamster ovary cell-derived recombinant human acid alpha-glucosidase in infantile-onset Pompe disease. $J$ Pediatr 2006; 149: $89-97$.

11 Okumiya T, Keulemans JL, Kroos MA et al: A new diagnostic assay for glycogen storage disease type II in mixed leukocytes. Mol Genet Metab 2006; 88: 22-28.

12 Okumiya T, Kroos M, Van Vliet L, Takeichi H, Van der Ploeg A, Reuser A: Chemical chaperones improve transport and enhance stability of mutant alpha- glucosidases in glycogen storage disease type II. Mol Genet Metab 2007; 90: 49-57.

13 Huie ML, Chen AS, Brooks SS, Grix A, Hirschhorn R: A de novo 13 nt deletion, a newly identified $\mathrm{C} 647 \mathrm{~W}$ missense mutation and a deletion of exon 18 in infantile onset glycogen storage disease type II (GSDII). Hum Mol Genet 1994; 3: $1081-1087$

14 Huie ML, Tsujino S, Brooks SS et al: Glycogen storage disease type II: identification of four novel missense mutations (D645N, G648S, R672W, R672Q) and two insertions/deletions in the acid $\alpha$-glucosidase locus of patients of differing phenotype. Biochem Biophys Res Commun 1998; 244: 921-927.

15 Kroos MA, Waitfield AE, Joosse M, Winchester B, Reuser AJ, MacDermot KD: A novel acid alpha-glucosidase mutation identified in a Pakistani family with glycogen storage disease type II. J Inherit Metab Dis 1997; 20: 556-558. 